\title{
Role of pinning in current driven domain wall motion in wires with perpendicular anisotropy
}

Cite as: Appl. Phys. Lett. 93, 172513 (2008); https://doi.org/10.1063/1.2998393

Submitted: 03 July 2008 . Accepted: 18 September 2008 . Published Online: 30 October 2008

C. Burrowes, D. Ravelosona, C. Chappert, S. Mangin, Eric E. Fullerton, J. A. Katine, and B. D. Terris

\section{ARTICLES YOU MAY BE INTERESTED IN}

Current-induced domain wall motion in a nanowire with perpendicular magnetic anisotropy

Applied Physics Letters 92, 202508 (2008); https://doi.org/10.1063/1.2926664

High domain wall velocities induced by current in ultrathin $\mathrm{Pt} / \mathrm{Co} / \mathrm{AlOx}$ wires with perpendicular magnetic anisotropy

Applied Physics Letters 93, 262504 (2008); https://doi.org/10.1063/1.3062855

Current-induced domain wall motion in perpendicularly magnetized CoFeB nanowire Applied Physics Letters 98, 082504 (2011); https://doi.org/10.1063/1.3558917

\section{Applied Physics Reviews} Now accepting original research 


\title{
Role of pinning in current driven domain wall motion in wires with perpendicular anisotropy
}

\author{
C. Burrowes, ${ }^{1}$ D. Ravelosona, ${ }^{1, a)}$ C. Chappert, ${ }^{1}$ S. Mangin, ${ }^{2}$ Eric E. Fullerton, ${ }^{3}$ \\ J. A. Katine, ${ }^{4}$ and B. D. Terris ${ }^{4}$ \\ ${ }^{1}$ Institut d'Electronique Fondamentale, UMR CNRS 8622, Université Paris Sud, \\ 91405 Orsay Cedex, France \\ ${ }^{2}$ LPM, U.H.P-Nancy I, B.P. 239 F-54506 Vandoeuvre Cedex, France \\ ${ }^{3}$ Center for Magnetic Recording Research,University of California, La Jolla, San Diego, \\ California 92093-0401, USA \\ ${ }^{4}$ San Jose Research Center, Hitachi Global Storage Technologies, 3403 Yerba Buena Road, \\ San Jose, California 95135, USA
}

(Received 3 July 2008; accepted 18 September 2008; published online 30 October 2008)

\begin{abstract}
Spatial and time resolved giant magnetoresistance measurements are used to detect thermally activated domain wall (DW) propagation along wires formed from spin valves having perpendicular magnetic anisotropy. We show that field- and current-induced DW propagation are both strongly influenced by the presence of pinning defects. As a result, the creep motion of DWs over macroscopic length scales is found to be stochastic. Only the combination of a high polarized current density and/or magnetic field allows DWs to propagate freely. These results may help explain low current-driven DW velocities observed in previous experiments. (C) 2008 American Institute of Physics. [DOI: 10.1063/1.2998393]
\end{abstract}

Renewed interest in the dynamics of domain walls (DWs) in nanostructures has been stimulated by the recent demonstration of current-induced DW motion in magnetic nanowires. ${ }^{1}$ The performance of such nanowires for low current applications such as magnetic memory and logic devices depends on the ultimate speed of DW motion under spinpolarized current. Also, the possibility to induce DW motion over large distances is crucial for the concept of race track memories $^{2}$ where multiple DWs are to be moved along the same wire. However, as the size of nanomagnets continues to decrease, an important factor affecting the dynamics and reliability of DW motion is the presence of pinning defects, ${ }^{3}$ whether resulting from structural defects present in the asgrown films or lithographic defects (e.g., edge roughness) originating from the patterning process. To gain a better understanding of the influence of defects on current induced domain wall motion requires techniques that spatially track the DW during its propagation along magnetic tracks. Exploiting the giant magnetoresistance (GMR) effect is one useful approach since it delivers an electrical signal, which is directly proportional to the position of a DW in one of the layers along a wire. Up to now, the GMR effect in wires has only been used in spin valves having in-plane magnetization to determine DW velocity under magnetic field ${ }^{4}$ or to measure current induced DW depinning from a pinning site. ${ }^{5,6}$

In this letter, we use the GMR effect to spatially track thermally activated DW motion in wires made from spin valves having perpendicular magnetic anisotropy (PMA). These systems are promising for applications in high density solid state memories. In the present samples we show that a DW undergoes many pinning events with different strength when propagating under magnetic fields and/or polarized currents. This gives rise to a stochastic and unreliable control of the DW motion, which could help explain reported DW

\footnotetext{
${ }^{\text {a) }}$ Author to whom correspondence should be addressed. Electronic mail: dafiné.ravelosona@u-psud.fr.
}

velocities under the action of current ${ }^{7-9}$ that are much lower than the maximum DW velocity allowed by spin angular momentum transfer rates.

Samples used in this study are spin valve structures based on $\mathrm{Co} / \mathrm{Ni}$ multilayers with PMA. ${ }^{10}$ The structure consists of a Pt/reference layer/Cu/free layer/Pt multilayer film. The reference layer is a composite $\left[(\mathrm{Co} / \mathrm{Pt})_{4} /(\mathrm{Co} / \mathrm{Ni})_{2}\right]$ to enhance the PMA $\left(5 \times 10^{6} \mathrm{erg} / \mathrm{cm}^{3}\right)$, while the free layer is a single $(\mathrm{Co} / \mathrm{Ni})_{4}$ film exhibiting a somewhat lower PMA. The sample growth procedure was the same as described in Ref. 10. High quality $100 \mu \mathrm{m}$ long and $0.2-1 \mu \mathrm{m}$ wide wires with low edge roughness $(<10 \mathrm{~nm})$ as shown in Fig. 1 were fabricated by means of electron beam lithography and ion milling. Along the wire there is a series of Hall crosses and gold contacts with a $d=40 \mu \mathrm{m}$ spacing. A large magnetic area at the end of the magnetic wires acts as a DW reservoir and is used to inject a single DW into the wire. Using this geometry, single DW motion in the free layer can be detected via both the GMR by measuring the longitudinal resistance between 2 contacts or the extraordinary Hall effect (EHE) by measuring the transverse voltage at Hall crosses. The present samples exhibit PMA with much higher GMR ratios up to $1.5 \%$ compared to previous $\mathrm{Co} / \mathrm{Pt}$ multilayers. ${ }^{10-12}$ Such a high GMR ration provides a spatial sensitivity down to $500 \mathrm{~nm}$ using a high sensitivity ac $(10 \mu \mathrm{A})$ lock in technique, which allows the detection of DW motion over large scale. The time resolution in this experiment was limited to $100 \mathrm{~ms}$.

Measurements of GMR as a function of time allows the determination of DW motion along the magnetic wire. This is illustrated in Fig. 2(a) for a $200 \mathrm{~nm}$ wide nanowire in a perpendicular applied field $H=280$ Oe. The initial deviation from the upper resistance level corresponds to the time needed by the DW to nucleate in the reservoir and reach the first electrical contact $\left[V_{-}\right.$in Fig. 1(a)]. The lowest resistance is reached while the DW is passing the second electrical contact. The variation in the GMR versus time corresponds 

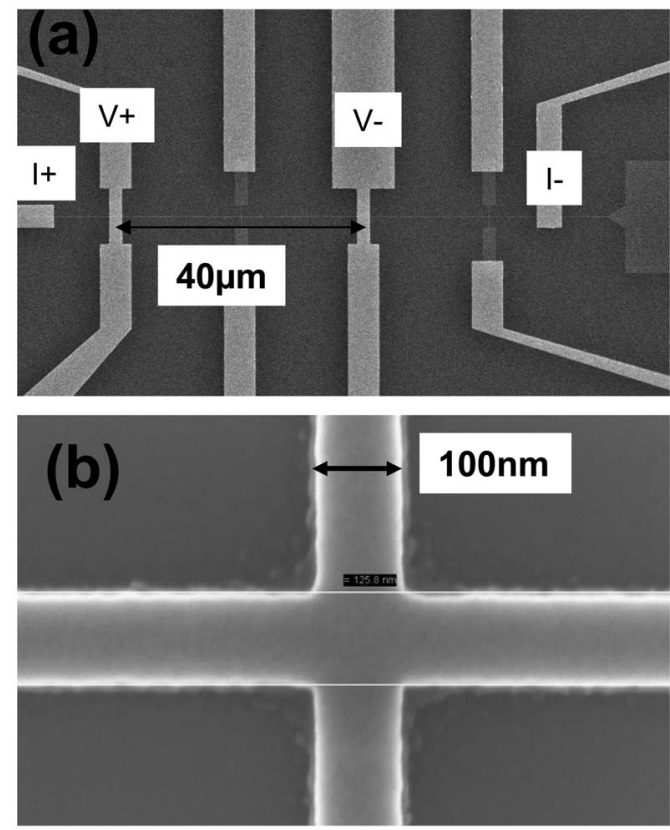

FIG. 1. (a) Scanning electron microscopy image of the magneto-transport device used in the experiments. The current is injected between electrodes $I_{+}$ and $I_{-}$and the DW motion is detected by means of the GMR effect between electrodes $V_{+}$and $V_{-}$that are $40 \mu \mathrm{m}$ apart. (b) The typical edge roughness in our devices is below $10 \mathrm{~nm}$ as seen in the high resolution image.

to a DW motion between the two $\mathrm{Au}$ contacts that are $40 \mu \mathrm{m}$ apart. We clearly observe that the DW motion occurs in a series of abrupt jumps separated by long plateaus where the DW is stationary. These features are consistent with the presence of strong random pinning sites that interact with the DW during its propagation in the wire. The abrupt jumps correspond to depinning of the DW from a pinning site and easy DW motion to the next pinning event. Note that the Hall cross seen in Fig. 1(a) between contacts $V_{+}$and $V_{-}$can induce pinning but the strength is much lower than the random pinning sites. Such a chaotic motion of a DW has been observed for magnetic fields ranging from $H=220$ Oe to $H$ $=320$ Oe. From these measurements, one can deduce an average DW velocity $v_{1}(H)$ by first taking into account the abrupt jumps only, which correspond to some portions of the wire where magnetization reversal takes place by easier DW motion. As shown in Fig. 2(a), the velocity data versus field are consistent with the expectations of a thermally activated regime given by $v_{1}(H)=v_{0} \exp \left[-\beta E_{1}\left(H, H_{p 1}\right)\right]$, where $\beta$ $=1 / k T, E\left(H, H_{p 1}\right)=U_{c 1}\left(H_{p 1} / H\right)^{-1 / 4}, H_{p 1}$ is the propagation field and $U_{c 1}$ is an energy constant in the wire. The exponent $1 / 4$, which was previously observed in similar wires, ${ }^{13}$ is expected in the case of a one dimensional interface moving in a transverse direction. Now, let us consider the field dependence of the time to traverse the wire that includes the duration of pinning events $\tau(H)$. One can also deduce an average DW velocity $v_{2}(H)=\tau(H) / d$ as illustrated in Fig. $2(\mathrm{a})$, which is obviously much lower than $v_{1}(H)$. In this case again, the average DW velocity $v_{2}(H)$ can be described by a creep behavior given by $v_{2}(H)=v_{0} \exp \left[-\beta E_{2}\left(H, H_{p 2}\right)\right]$, where $E\left(H, H_{p 2}\right)=U_{c 2}\left(H_{p 2} / H\right)^{-1 / 4}$ and $H_{p 2} \gg H_{p 1}$.

These features are consistent with the DW motion/ depinning being in a thermally activated creep regime. However, note that the distribution of jumps and plateaus are an indication of a large distribution of propagation fields $H_{p}$

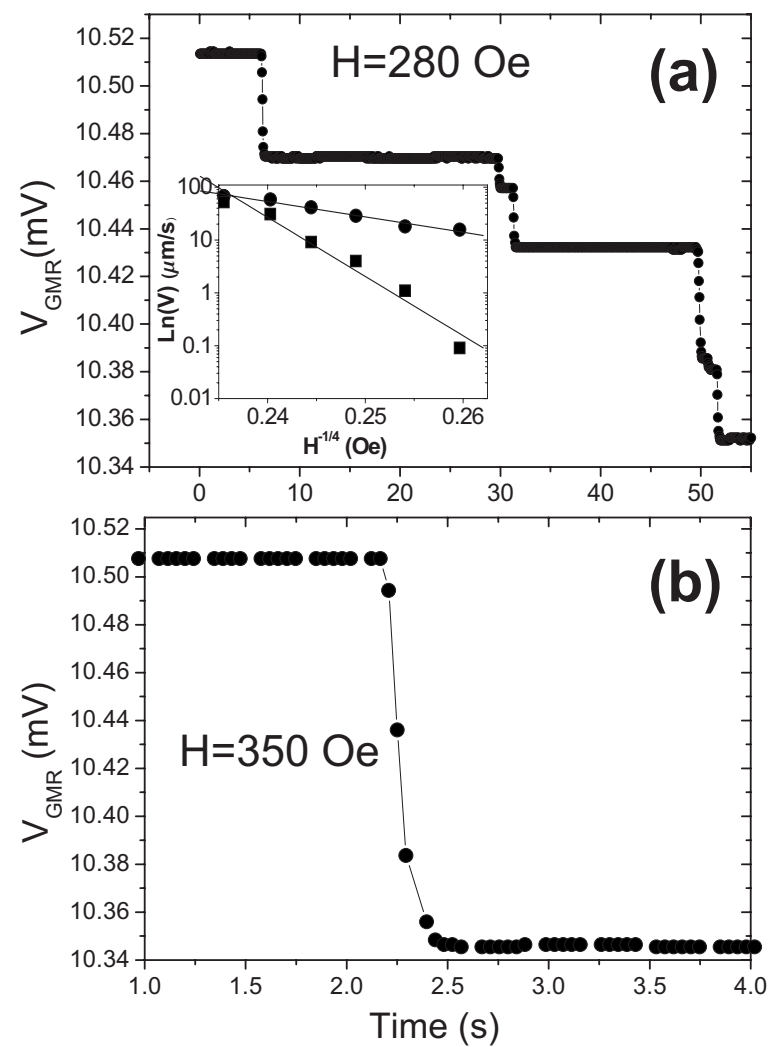

FIG. 2. (a) Measurement of GMR as a function of time in a 200-nm wide wire for magnetic fields of $H=280$ Oe. The inset corresponds to the average DW velocity measured by taking into account only the duration of jumps (circles) and including the duration of plateaus (squares). (b) Same as (a) but for $H=350$ Oe. The plateaus correspond to a few DW pinning events on local defects along the $40-\mu \mathrm{m}$ long wire.

along the wires. The creep motion described by $v_{1}(H)$ and $v_{2}(H)$ do not fully capture this case where $H_{p 1}\left(H_{p 2}\right)$ corresponds to an average of the lowest (highest) part of the distribution. Finally, note that the pinning events seen in Fig. 2(a) have been observed for more than ten different wires ranging from $200 \mathrm{~nm}$ to $1 \mu \mathrm{m}$ in size. In all cases, the typical field to achieve steady DW motion is about 320 Oe. Figure 2(b) shows the corresponding GMR versus time for an applied field of $H=350$ Oe. The variation in the GMR between roughly 2.2 and $2.5 \mathrm{~s}$ corresponds to a steady DW motion giving rise to an average DW velocity of $\sim 200 \mu \mathrm{m} / \mathrm{s}$. This again corresponds to thermally activated DW motion since a viscous regime is expected for DW velocity in the range of a few $\mathrm{m} / \mathrm{s}^{3}$. However, because our time resolution is limited to $100 \mathrm{~ms}$, the flow regime observed in Fig. 2(b) as well as the abrupt jumps observed in Fig. 2(a) may correspond again to a motion dominated by pinning events times shorter than $100 \mathrm{~ms}$ rather than a continuous motion.

In order to study the spin transfer effect, a DW is first driven to a rest position in the wire between the two longitudinal contacts by field and then the current is increased while measuring the GMR effect. However, using critical current densities up to $10^{8} \mathrm{~A} / \mathrm{cm}^{2}$ it was not possible to detect any current induced DW motion at zero fields on a scale higher than the spatial resolution of our technique $(\sim 500 \mathrm{~nm})$, except in a very few irreproducible cases. In films with PMA, we have previously ${ }^{10}$ shown that the critical current density to move a DW is proportional to the pinning force, which is consistent with the presence of a nonadiabatic 

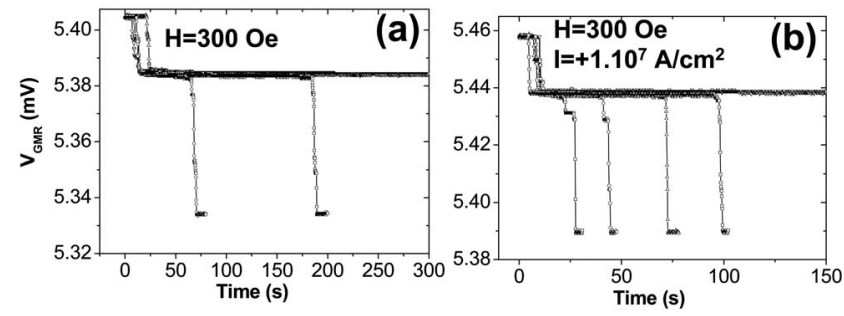

FIG. 3. Five measurements of DW motion in a $500 \mathrm{~nm}$ wide wire at fixed fields and currents (a) $H=300 \mathrm{Oe}, J=0$ and (b) $H=300 \mathrm{Oe}, J=$ $+1.10^{7} \mathrm{~A} / \mathrm{cm}^{2}$. A positive current acts in the same direction as a positive magnetic field.

spin-transfer term. ${ }^{14}$ Thus a distribution of $H_{p}$ along the wires may induce a distribution of threshold currents. As the DW is first initialized at (plateau) position in the wire corresponding to the strong pinning sites, it is not possible to move it reproducibly from this site using only a polarized current.

Shown in Figs. 3(a) and 3(b) are multiple measurements of DW motion at fixed fields and current density $(H$ $=300$ Oe and $J=0)$ and $\left(H=300\right.$ Oe and $\left.J=1.10^{7} \mathrm{~A} / \mathrm{cm}^{2}\right)$ respectively, for a $500 \mathrm{~nm}$ wide wire. The presence of pinning centers combined with thermal activation induces a dynamical behavior that is not reproducible. Particularly, the distribution of pinning times from the same pinning position is very large, ${ }^{15}$ which induces a stochastic behavior along the wire. We observed [Fig. 3(b)] that the application of a polarized current $\left(\sim 10^{7} \mathrm{~A} / \mathrm{cm}^{2}\right)$ that acts in the same direction than the magnetic field reduces the average time for the DW to propagate. This has been confirmed by statistical measurements on a single pinning event ${ }^{15}$ that show a shift in the distribution of pinning times to lower times and a reduction in the width of the distribution. However, even for a combination of such high current and magnetic field, the motion of the DW is still irreproducible. Only current densities of $5 \times 10^{7} \mathrm{~A} / \mathrm{cm}^{2},{ }^{16}$ combined with high magnetic fields $H$ $=230$ Oe results in DW propagation without any pinning along the $40 \mu \mathrm{m}$ wire [Fig. 4(a)]. Reducing the current [Fig. 4(b)] results in the emergence of DW pinning.

An important result of our study is that from these measurements, it is then not possible to evaluate accurately the DW velocity under fields and/or currents since it is dominated by the pinning times leading to a stochastic process. This holds for the thermally activated regimes below $H_{p}$ as well as the viscous regime above $H_{p}$ where a single pinning event can dominate the propagation. These observations could explain reported DW velocities under current that are much lower than the maximum DW velocity allowed by the spin angular momentum transfer rate. ${ }^{7-9}$ Only recent studies using very high quality wires ${ }^{1,17}$ where the effects of DW pinning is minimized have found velocities expected from spin transfer.

One important point is the origin of such pinning centers. One possible origin is linked to intrinsic nanometric defects from the as-grown films, such as surface roughness, boundaries between crystallites, and atomic steps at interfaces or dislocations. The typical length scale (distance between defects) for such a pinning potential corresponds to a few tens of nanometers (typical lateral size of grains and terraces). ${ }^{3}$ Also, edge roughness [see Fig. 1(b)] due to the patterning process can affect locally the propagation of the
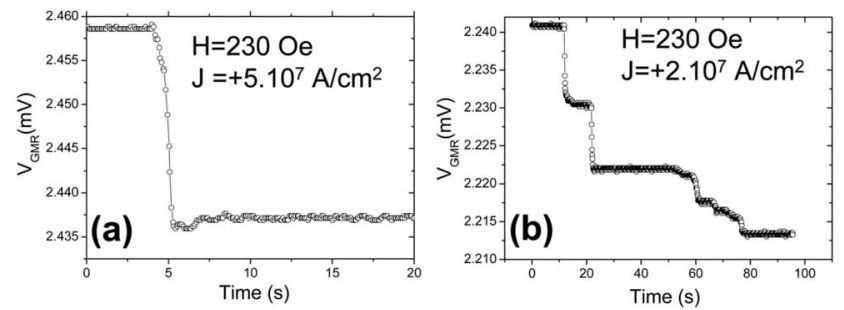

FIG. 4. Measurement of GMR as a function of time in a $1-\mu \mathrm{m}$ wide wire for different values of field and current density (a) $H=230 \mathrm{Oe}, J=5$ $\times 10^{7} \mathrm{~A} / \mathrm{cm}^{2}$, and (b) $H=200 \mathrm{Oe}, J=5 \times 10^{7} \mathrm{~A} / \mathrm{cm}^{2}$.

DW as we have shown in a previous study. ${ }^{13}$ However, we have not observed any increase in the pinning strength when decreasing the wire width from $1 \mu \mathrm{m}$ to $200 \mathrm{~nm}$, which indicates that edges do not play an important role here.

In conclusion, we have shown that the presence of a few strong pinning defects affect drastically the creep motion of a single DW under magnetic field and/or polarized currents along wires with PMA. This study shows that in order not to misinterpret the data, it is crucial to use a detection technique that is able to track spatially a DW during its motion. In addition, the stochastic nature of thermally activated phenomena requires statistical studies to quantify currentinduced DW motion. Finally, this study highlights that DW motion tracked by GMR measurements is a powerful tool to probe the pinning potential on a nanometric scale.

This work was supported in part by the contract ISTRADE of the French National Research Agency (ANR) and by the contract "MAGLOG" of C'NANO Ile de France through a Ph.D. grant.

${ }^{1}$ See S. S. Parkin, M. Hayashi, and L. Thomas, Science 320, 190 (2008), and references therein.

${ }^{2}$ M. Hayashi, L. Thomas, R. Moriya, C. Rettner, and S. S. Parkin, Science 320, 209 (2008).

${ }^{3}$ J. Ferre, Spin Dynamics in Confined Magnetic Structures I (Springer, New York, 2002), Vol. 83, p. 127.

${ }^{4}$ T. Ono, H. Miyajima, K. Shigeto, K. Mibu, N. Hosoito, and T. Shinjo, Science 284, 468 (1999).

${ }^{5}$ J. Grollier, P. Boulenc, V. Cros, A. Hamzic, A. Vaurs, A. Fert, and G. Faini, Appl. Phys. Lett. 83, 509 (2003).

${ }^{6}$ S. Laribi, V. Cros, M. Muñoz, J. Grollier, A. Hamzić, C. Deranlot, A. Fert, and E. Martinez, Appl. Phys. Lett. 90, 232505 (2007).

${ }^{7}$ A. Yamaguchi, T. Ono, S. Nasu, K. Miyake, K. Mibu, and T. Shinjo, Phys. Rev. Lett. 92, 077205 (2004).

${ }^{8}$ M. Kläui, P.-O. Jubert, R. Allenspach, A. Bischof, J. A. C. Bland, G. Faini, U. Rüdiger, C. A. F. Vaz, L. Vila, and C. Vouille, Phys. Rev. Lett. 95, 026601 (2005).

${ }^{9}$ M. Klaui, M. Laufenberg, L. Heyne, D. Backes, U. Rudiger, C. A. F. Vaz, J. A. C. Bland, L. J. Heyderman, S. Cherifi, A. Locatelli, T. O. Mentes, and L. Aballe, Appl. Phys. Lett. 88, 232507 (2006).

${ }^{10}$ D. Ravelosona, S. Mangin, J. A. Katine, E. E. Fullerton, and B. D. Terris, Appl. Phys. Lett. 90, 072508 (2007).

${ }^{11}$ D. Ravelosona, D. Lacour, J. A. Katine, B. D. Terris, and C. Chappert, Phys. Rev. Lett. 95, 117203 (2005).

${ }^{12}$ S. Mangin, D. Ravelosona, J. Katine, B. Terris, and E. E. Fullerton, Nat. Mater. 5, 210 (2006).

${ }^{13}$ F. Cayssol, D. Ravelosona, C. Chappert, J. Ferré, and J. P. Jamet, Phys. Rev. Lett. 92, 107202 (2004).

${ }^{14}$ A. Thiaville, Y. Nakatani, J. Miltat, and Y. Susuki, Europhys. Lett. 69, 990 (2005).

${ }^{15}$ C. Burrowes, A. P. Mihai, J. P. Attane, L. Vila, A. Marty, C. Chappert, I Tudosa, E. E. Fullerton, and D. Ravelosona, Nature Mater. (submitted).

${ }^{16}$ The estimated heating of the wire from a current density of 5 $\times 10^{7} \mathrm{~A} / \mathrm{cm}^{2}$ is $\sim 100^{\circ} \mathrm{C}$.

${ }^{17}$ M. Hayashi, L. Thomas, C. Rettner, R. Moriya, Y. B. Bazaliy, and S. S. P. Parkin, Phys. Rev. Lett. 98, 037204 (2007). 